\title{
POTENCIAL BIOTECNOLÓGICO PARA LA PRODUCCIÓN SUSTENTABLE DE BIODIESEL DE MICROALGAS OLEAGINOSAS AISLADAS DEL RÍO ITAYA, LORETO, PERÚ
}

\section{BIOTECHNOLOGICAL POTENTIAL FOR SUSTAINABLE BIODIESEL PRODUCTION OF OLEAGINOUS MICROALGAE ISOLATED FROM ITAYA RIVER, LORETO, PERU}

\author{
Marianela Cobos Ruiz¹, Juan Carlos Castro Gómez², y Luis Alexander Cerdeira Gutierrez²
}

\section{Resumen}

Las microalgas son una buena alternativa para producir biodiesel, pero la falta de cepas ideales ha impedido la viabilidad económica de estos sistemas de producción. El objetivo fue aislar e identificar microalgas oleaginosas con potencial aplicación para la producción sustentable de biodiesel. Las muestras fueron colectadas del río Itaya y enriquecidas con medio CHU. Las microalgas oleaginosas se identificaron y aislaron con técnicas estándares. Los lípidos totales se extrajeron con solventes orgánicos y analizaron mediante HPLC-DAD. Se han identificado 24 especies microalgales, de las cuales el 50\% fueron oleaginosas. De éstas, se aislaron Ankystrodesmus sp., Chlorella sp.y Scenedesmus sp., sobresaliendo Ankystrodesmus sp., por presentar $43.6 \%$ de lípidos totales con predominancia de triglicéridos. En conclusión, varias de las especies microalgales del río Itaya son oleaginosas que deben ser evaluadas para determinar si son promisorias para la producción de biodiesel. Asimismo, las tres especies de microalgas oleaginosas aisladas muestran diferencias en sus perfiles de crecimiento, en la cantidad de biomasa y en la cantidad y tipos de lípidos totales. Ankystrodesmus sp. es la especie más interesante por tener un alto contenido de lípidos totales $(>40 \%)$ con predominancia de triglicéridos, por tanto es una microalga oleaginosa promisoria para la producción sustentable de biodiesel.

Palabras clave: Amazonía, biocombustibles, biotecnología, triglicéridos.

\begin{abstract}
Microalgae are a great alternative in biodiesel production, but the lack of ideal strains has impeded the economic viability in these production systems. The objective of this work was to isolate and identify oleaginous microalgae with potential applications for sustainable biodiesel production. Samples were collected from Itaya River and grown in a CHU medium. Oleaginous microalgae were identified and isolated by standard techniques. Total lipids were extracted with organic solvents and analyzed by HPLC-DAD. 24 microalgal species, of which 50\% were oleaginous, were identified. Of these, we isolated Ankystrodesmus sp., Chlorella sp. and Scenedesmus sp., were isolated as well as Ankystrodesmus sp., which stands out because it has $43.6 \%$ of total lipids, predominantly triglycerides. In conclusion, several of the Itaya River microalgae species are oleaginous that must be evaluated to determine if they are promising for biodiesel production. Also, the three oleaginous microalgae species isolated show differences in their growth profiles, in the amount of biomass and in the amount and type of fatty acids profile. Ankystrodesmus sp. is the most interesting species, having a high total lipid content ( $>40 \%)$ with a predominance of triglycerides. It is therefore, a promising oleaginous microalgae for sustainable biodiesel production.
\end{abstract}

Key words: Amazonia, biofuels, biotechnology, triglycerides.

\section{Introducción.}

La creciente demanda energética mundial, el agotamiento y elevado costo de los combustibles fósiles y el cambio climático son problemas que deben ser solucionados con urgencia (Edenhofer et al., 2012). Para mitigar estos problemas, nuestro país cuenta con propuestas de política de estado para ampliar la matriz energética utilizando fuentes renovables. Estas medidas legales han incrementado la importación de biodiesel por la insignificante producción nacional. El Biodiesel es un biocombustible que está constituido por ésteres de metilo de ácidos grasos de cadena larga derivado de diversos tipos de aceite de origen animal, vegetal e incluso de microalgas, que debe cumplir con ciertas especificaciones técnicas para ser usado en motores 
diesel (Menetrez, 2012). Típicamente, el biodiesel es producido a partir de una reacción química denominada transesterificación, la cual se logra incubando a $70^{\circ} \mathrm{C}$ una mezcla de lípidos, metanol y un catalizador básico (Lam \& Lee, 2012).

En nuestro país la producción de biodiesel depende primariamente del cultivo de grandes extensiones de palma aceitera y piñón blanco (Veen \& Carrillo, 2009). La utilización de grandes superficies de monocultivos y el cambio en el uso de la tierra arriesgamos la seguridad alimentaria y podemos provocar la pérdida de la biodiversidad y de los servicios ecosistémicos (Delucchi, 2010). Por tanto, es imperioso disponer de alternativas bioenergéticas más adecuadas. Una buena opción son las microalgas oleaginosas, por su alta eficiencia fotosintética comparada con plantas terrestres $>7 \%$ (Georgianna \& Mayfield, 2012; Sharma et al., 2012), algunas especies microalgales presentan productividades de 10 a 100 veces mayores que los cultivos convencionales de plantas terrestres, (Garibay et al., 2009; Loera \& Olguín, 2009) actúan como sumideros de $\mathrm{CO}_{2}$ (Ho et al., 2011) y pueden utilizar aguas servidas como medio para la producción de biomasa que posteriormente se utilizará como materia prima en forma de lípidos para producir biodiesel ( $\mathrm{Wu}$ et al., 2012; Zhou et al., 2011). Sin embargo, aún existen varios obstáculos tecnológicos y económicos que deben solucionarse (Khoo et al., 2011) y uno de los mayores retos es el aislamiento, selección y cultivo de microalgas robustas que presenten óptimo contenido de triglicéridos, posean altas tasas de crecimiento y sean resistentes a diferentes tipos de contaminación (Duong et al., 2012; Larkum et al., 2012; Sheehan et al., 1998; Wijffels \& Barbosa, 2010).

Los estudios sobre microalgas amazónicas realizados a la fecha, sólo se han limitado a su identificación taxonómica, (López, 2009; Sánchez, 1991; Cobos et al., 2012) no existiendo reportes de aislamientos y de evaluaciones del potencial biotecnológico para la producción de biodiesel. Por tanto, el objetivo de esta investigación fue aislar e identificar microalgas oleaginosas amazónicas con potencial aplicación para la producción sustentable de biodiesel.

\section{Materiales y métodos.}

Colecta, enriquecimiento e identificación de microalgas.

Las muestras de agua $(100 \mathrm{ml})$ fueron colectadas en el río Itaya (0695334 y 9585835 UTM) en época de creciente (10/Mar/2013). Este río está ubicado en la provincia de Maynas, Departamento de Loreto, Perú. Las muestras fueron colocadas en frascos oscuros (para protegerlos de la luz), sellados herméticamente y transportadas en termos (a una temperatura promedio de $18^{\circ} \mathrm{C}$ ) al laboratorio de Biotecnología y Bioenergética de la Universidad Científica del Perú.
Posteriormente, $5 \mathrm{ml}$ de cada muestra fue inoculada en $50 \mathrm{ml}$ de medio CHU (Barclay et al., 1986) el cual está constituido por $0.032 \mathrm{mM}$ de $\mathrm{KH}_{2} \mathrm{PO}_{4}, 0.101 \mathrm{mM}$ de $\mathrm{MgSO}_{4} .7 \mathrm{H}_{2} \mathrm{O}, 0.250 \mathrm{mM}$ de $\mathrm{Ca}\left(\mathrm{NO}_{3}\right)_{2} .3 \mathrm{H}_{2} \mathrm{O}, 0.188$ $\mathrm{mM}$ de $\mathrm{NaHCO}_{3}, 0.009 \mathrm{mM}$ de $\mathrm{FeCl} 3.6 \mathrm{H}_{2} \mathrm{O}, 0.010$ $\mathrm{mM}$ de Na-EDTA, $11.5 \mu \mathrm{M}$ de $\mathrm{H}_{3} \mathrm{BO}_{3}, 0.2 \mu \mathrm{M}$ de $\mathrm{MnCl}_{2} .4 \mathrm{H}_{2} \mathrm{O}, 0.2 \mu \mathrm{M}$ de $\mathrm{ZnSO}_{4} .7 \mathrm{H}_{2} \mathrm{O}, 0.03 \mu \mathrm{M}$ de $\mathrm{Na}_{2} \mathrm{MoO}_{4} .2 \mathrm{H}_{2} \mathrm{O}, 0.08 \mu \mathrm{M}$ de $\mathrm{CuSO}_{4} .5 \mathrm{H}_{2} \mathrm{O}$, y $0.04 \mu \mathrm{M}$ de $\mathrm{CoSO}_{4} .7 \mathrm{H}_{2} \mathrm{O}$. El homogenizado fue transferido a un frasco Erlenmeyer para su cultivo en agitación (110 $\mathrm{rpm}$ ), a $28^{\circ} \mathrm{C}$ y expuesto a luz de fluorescentes con una intensidad de $20 \mu \mathrm{mol} . \mathrm{m}^{-2} . \mathrm{s}^{-1}$ por cuatro semanas hasta observar cambio en la coloración del medio de cultivo. Para verificar la presencia de las microalgas de interés se utilizó alícuotas $(100 \mu 1)$ del cultivo y se observó en microscópico a 40X (Carl ZeissAxioLab.A1). La identificación taxonómica se realizó utilizando claves taxonómicas de Bicudo \& Menezes (2006).

Aislamiento, cultivo y cosecha de las microalgas.

El aislamiento se llevó a cabo empleando la técnica estándar de lavado celular por aislamiento con pipeta capilar (Arredondo \& Vázquez, 1991). Consistió en colocar una gota del cultivo en una lámina portaobjeto y seis gotas de medio CHU. Luego con una pipeta capilar conectada a una manguerilla de silicona con filtro se succionó y transfirió las microalgas de interés hacia la primera gota del medio de cultivo. Este proceso de transferencia se repitió hasta obtener una célula microalgal sin contaminación evidente. Posteriormente, la gota con microalga fue transferida a un tubo de ensayo con $2 \mathrm{ml}$ de medio CHU. Luego las células microalgales fueron cultivadas en las condiciones indicadas anteriormente (agitación a $110 \mathrm{rpm}, 28^{\circ} \mathrm{C}$ y $20 \mu \mathrm{mol} \cdot \mathrm{m}^{-2} \cdot \mathrm{s}^{-1}$ ) durante 2 a 3 semanas hasta observar la pigmentación del medio. La pureza del cultivo se evaluó mediante análisis microscópico. Los cultivos unialgales fueron transferidos gradualmente a matraces Erlenmeyer conteniendo $125,250,500$ y $1000 \mathrm{ml}$ de medio de cultivo. La tasa del crecimiento celular de los cultivos unialgales se evaluó cada 24 horas. Para ello, $100 \mu 1$ del cultivo se homogenizó con $50 \mu \mathrm{l}$ de lugol y se transfirió a un hematocitómetro para el recuento celular con un microscopio a 40x (Carl ZeissAxioLab.A1). Los cultivos y recuentos celulares se realizaron por triplicado. La cosecha de las microalgas se realizó por centrifugación del cultivo a $1730 \mathrm{~g} * 10$ ' a $4{ }^{\circ} \mathrm{C}$. El precipitado microalgal obtenido fue transferido a placas petri y se dejó secar a $50^{\circ} \mathrm{C}$ por 24 horas.

Análisis microscópico de microalgas oleaginosas.

Para determinar la presencia de los triglicéridos intracelulares de las microalgas oleaginosas aisladas se utilizó una técnica de tinción mediante el fluorocromo Rojo Nilo (Greenspan \& Fowler, 1985). Para ello, las células fueron teñidas con $2 \mu \mathrm{g} / \mathrm{ml}$ de Rojo Nilo (disuelto en acetona) por 15 minutos en 
oscuridad y fotografiadas empleando un microscopio trinocular de epifluorescencia Carl Zeiss-AxioLab.A1 y una cámara digital AxioCamERc 5s en tiempo real. Las imágenes fueron obtenidas con una magnificación de $100 \mathrm{X}$ con luz visible y epifluorescencia (excitación: 510-560, emisión: 590).

Extracción de lípidos totales.

Se realizó de acuerdo a Yu et al., (2012). $50 \mathrm{mg}$ de microalgas secas fueron transferidos y triturados a un mortero con $8 \mathrm{ml}$ de cloroformo:metanol (2:1). El extracto obtenido se transfirió a microtubos de $2 \mathrm{ml}$ y se les añadió $100 \mu \mathrm{l}$ de $\mathrm{NaCl} 0.9 \%$ por cada $1000 \mu \mathrm{l}$ de extracto. La solución se homogenizó en vortex por 30 segundos y centrifugó a $13000 \mathrm{~g} * 5^{\prime}$ a $4^{\circ} \mathrm{C}$ La fase clorofórmica se filtró con filtros para jeringa de 0.25 $\mu \mathrm{m}$ y se transfirió a vasos de precipitado de peso conocido para evaporar el cloroformo en un hotplate a $50^{\circ} \mathrm{C}$ por un tiempo de 4 horas. Luego los componentes lipídicos retenidos en el vaso fueron secados a $50^{\circ} \mathrm{C}$ por 4 horas y se atemperó a $25^{\circ} \mathrm{C}$ para determinar su peso. Por diferencia de peso del vaso de precipitado con y sin los lípidos se determinó la cantidad de lípidos totales obtenidos, con la siguiente ecuación:

Contenido de lípidos $(\%)=\left(P_{L} / P_{M}\right) \times 100$,

Donde $P_{L}$ es el peso seco de los lípidos totales y $P_{M}$ es el peso seco de las microalgas.

Análisis cromatográfico de lípidos totales.

Fue realizado de acuerdo a De La Mata (2011), con modificaciones en el sistema de detección empleado. Los lípidos totales obtenidos fueron disueltos con $2 \mathrm{ml}$ de $\mathrm{n}$ hexano:isopropanol (1:1). La solución lipídica fue diluida 10 veces $(100 \mu \mathrm{l}$ de solución lipídica $+900 \mu \mathrm{l}$ de $\mathrm{n}$ hexano:isopropanol) para realizar su análisis mediante cromatografía líquida de alta performancia con detector de arreglo de diodos (HPLC-DAD). El análisis cromatográfico se realizó empleando una columna Purospher ${ }^{2}$ STARLiChroCART RP-18e (5 $\mu \mathrm{m}, 250 \mathrm{x} \quad 4,6 \mu \mathrm{mm})$. La temperatura de la columna fue mantenida a $30^{\circ} \mathrm{C}$. El volumen de inyección de la muestra fue $10 \mu \mathrm{l}$. Se empleó una fase móvil binaria compuesta de acetonitrilo y n-hexano:isopropanol $(1: 1)$ y se usó una gradiente lineal de 60:40 a 45:55 en 40 minutos y un tiempo adicional de 5 minutos en la proporción 45:55. La velocidad de flujo de la fase móvil fue ajustada a $1 \mathrm{ml} / \mathrm{min}$. La detección de los lípidos totales se realizó de 210 a $800 \mathrm{~nm}$ y la detección de los triglicéridos a $215 \mathrm{~nm}$.
Análisis estadístico.

Se determinó el promedio y desviación estándar de la cantidad de biomasa y lípidos totales obtenidos de las especies aisladas. Para evaluar las diferencias en la cantidad promedio de biomasa y lípidos totales obtenidos se realizó el ANOVA y el test HSD de Tukey con un nivel de confianza del 95\% y considerando las diferencias estadísticas significativas cuando $\mathrm{P}<0.05$. Todos los análisis se hicieron con el programa IBM SPSS Statistics 21.

\section{Resultados y discusión.}

Después de enriquecer las muestras colectadas del río Itaya con el medio CHU y realizado el cultivo por cuatro semanas, se ha registrado 24 especies de microalgas pertenecientes a 15 familias y seis divisiones (Tabla 1). Los grupos taxonómicos en cuanto a división más representativos reportados para el presente estudio fueron: Bacillariophyta, Chlorophyta y Cyanophyta, estos resultados concuerdan con López (2009) quien reportó estas divisiones como las de mayor representatividad en un estudio sobre caracterización fitoplanctónica física y química de la Cocha Llanchama en los períodos hidrológicos de creciente y media vaciante. Sin embargo, Sánchez (1991) reportó que la división Chlorophyta fue la más representativa tanto en número de organismos, géneros y especies lo que también concuerda con los resultados obtenidos en este estudio.

Asimismo, del total de microalgas oleaginosas registradas, se aislaron y cultivaron exitosamente las

Tabla 1. Especies de microalgas identificadas en muestras obtenidas del río Itaya.

\begin{tabular}{|c|c|c|}
\hline División & Familia & Especie \\
\hline \multirow[t]{6}{*}{ Bacillariophyta } & Fragilariaceae & Fragilaria sp. \\
\hline & Amphipleuraceae & Frustulia rhomboide \\
\hline & & Frustulia vulgaris \\
\hline & Naviculaceae & Navicula radiosa* \\
\hline & & Navicula sp.* \\
\hline & Pinnulariaceae & Pinnularia sp. \\
\hline \multirow[t]{13}{*}{ Chlorophyta } & Chlorellaceae & Ankistrodesmus acicularis* \\
\hline & & Ankistrodesmus falcatus* \\
\hline & & Ankistrodesmus nannoselene* \\
\hline & & Ankistrodesmus sp.* \\
\hline & & Chlorella sp.* \\
\hline & Chlorococcaceae & Tetraedron sp. \\
\hline & Closteriaceae & Closterium sp. \\
\hline & Desmidiaceae & $\begin{array}{l}\text { Cosmarium sp. } \\
\text { Penium sp. }\end{array}$ \\
\hline & Oocystaceae & Closteriopsis acicularis \\
\hline & & Scenedesmus acuminatus* \\
\hline & & Scenedesmus linearis* \\
\hline & & Scenedesmus sp.* \\
\hline & Volvocaceae & Gonium pectorale \\
\hline Crysophyceae & Synuraceae & Mallomonas sp. \\
\hline Cyanophyta & Oscillatoriaceae & Oscillatoria sp. \\
\hline Euglenophyta & Euglenaceae & Euglena sp. \\
\hline Xantophyta & Characiopsidaceae & Characiopsis sp. \\
\hline
\end{tabular}

*: Microalgas oleaginosas 
especies Ankystrodesmus sp., Chlorella sp. y Scenedesmus sp. (Figura 1). Estas especies mostraron diferencias en sus curvas de crecimiento en el medio CHU. Chlorella sp. y Scenedesmus sp. presentaron mayores densidades celulares (concentración inicial $\sim 0.4 \times 10^{6}$, concentración final $4 \times 10^{6} \mathrm{cel} / \mathrm{ml}$ ) desde los primeros días de iniciado el cultivo, mientras que Ankystrodesmus sp., mostró un crecimiento lento en el inicio ( $0.04 \times 10^{6} \mathrm{cel} / \mathrm{ml}$ ) debido a la baja densidad celular y su crecimiento fue aumentando de manera gradual en función al tiempo de cultivo, con un incremento significativo a las dos semanas de cultivo (concentración final $2.3 \times 10^{6} \mathrm{cel} / \mathrm{ml}$ ). Las caídas en el crecimiento microalgal se deben principalmente a la adición de medio fresco lo cual diluye el cultivo. Se observó que cada especie de microalga respondió de manera diferente a las condiciones de cultivo. Además, la curva de crecimiento también depende del tiempo de cultivo y la adición de nutrientes al medio. Leonardos \& Lucas (2000); Brown et al. (1993) indicaron que el perfil de crecimiento y producción de lípidos de las microalgas puede ser alterado sustancialmente por la manipulación de las condiciones abióticas en el cultivo tales como intensidad de luz, fotoperiodo o temperatura, tal como se pudo evidenciar en el presente trabajo donde las microalgas alcanzaron densidades celulares bajas en las condiciones de cultivo anteriormente descritas, comparado con los resultados de Castillo et al., (2010) quienes obtuvieron valores superiores a los 26 millones de células/ml al cultivar Dunaliella salina bajo condiciones de salinidad de $35 \%$ con agitación continua y empleando el medio ( $\mathrm{J} / 1)$ modificado, pero

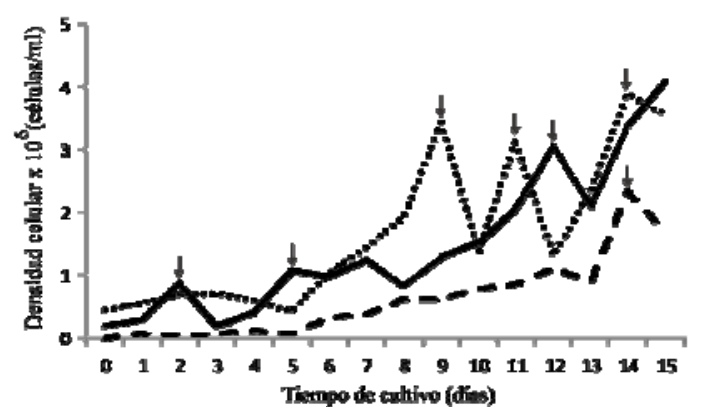

Figura 1. Perfil de crecimiento de las microalgas oleaginosas aisladas: Ankystrodesmus sp. (---), Chlorella sp. (...) y Scenedesmus sp. (—). Las flechas $(\downarrow)$ indican los momentos en que se añadieron más medio de cultivo CHU a los cultivos microalgales.

esta productividad en biomasa no fue suficiente para conseguir un elevado contenido de lípidos, lo que si obtuvieron con Chlorella nativa, la cual por medio de una alta velocidad de agitación por aireación y un valor de $\mathrm{pH}$ inicial de 7.5 lograron inducir un factor de estrés en su crecimiento, lo que se tradujo en una mayor producción de lípidos.

Las microalgas aisladas al ser teñidas con Rojo Nilo nos permitieron determinar diferencias cualitativas respecto a la presencia de lípidos en el interior de ellas aún sin ser inducidas, tal como se muestra en la Figura 2. Es evidente que las tres especies de microalgas acumularon triglicéridos. La tinción con Rojo Nilo puede servir como un colorante
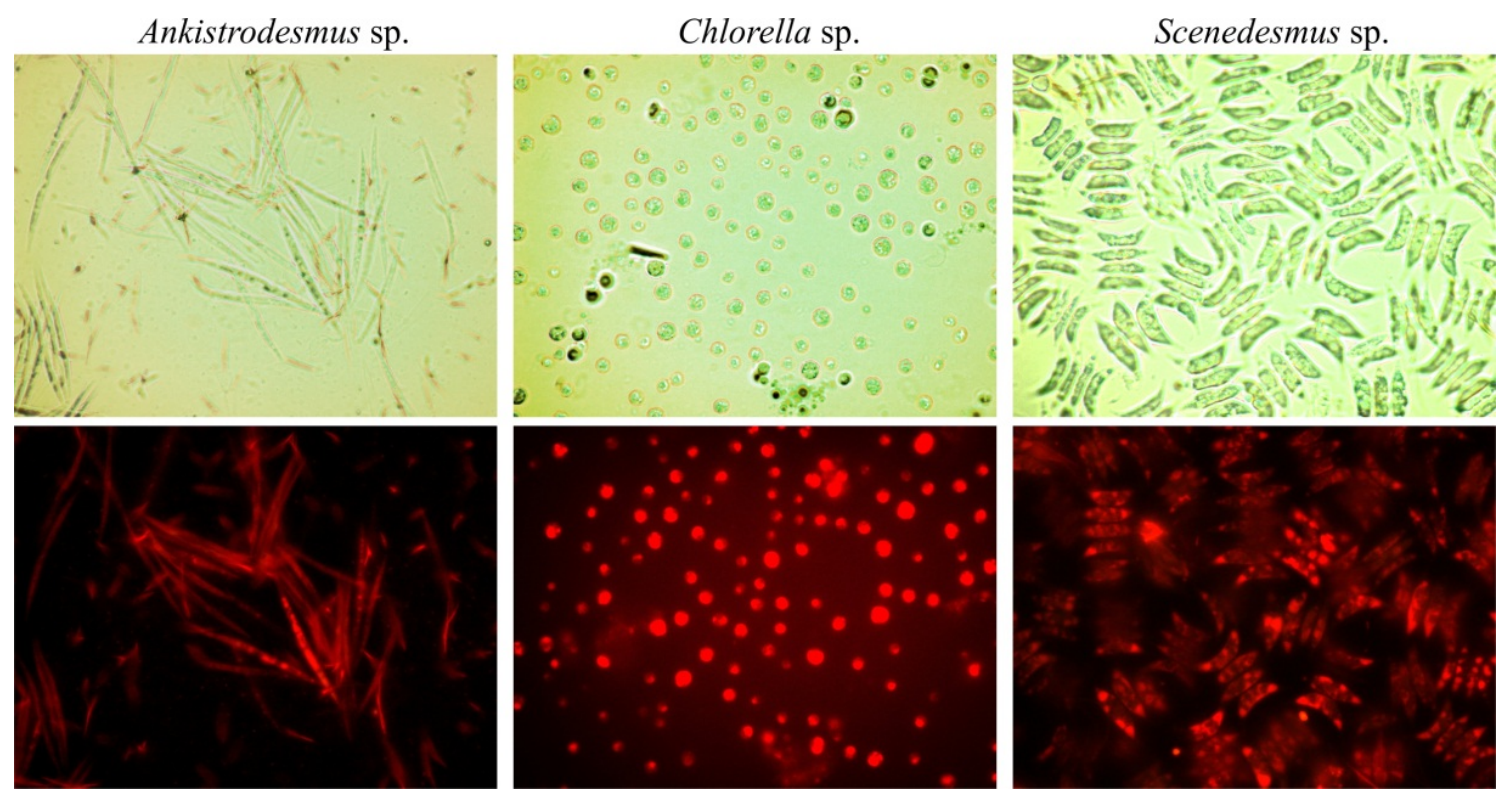

Figura 2. Microfotografías con luz visible y epifluorescencia de las tres especies de microalgas oleaginosas aisladas. 
sensible para la detección de cuerpos lipídicos citoplasmáticos (Greenspan et al. 1985) y es comúnmente usada para teñir lípidos en microorganismos como bacterias, levaduras y microalgas para determinar cualitativamente la presencia de lípidos en el interior de las células (Duong et al. 2012), lo cual se confirma en este estudio. En esta investigación, demostramos la utilidad de este tipo de tinción para la determinación cualitativa de cuerpos lipídicos citoplasmáticos en las microalgas oleaginosas amazónicas. Esta técnica de ahorro de tiempo ofrece una alternativa a los protocolos de cuantificación de lípidos basados en gravimétricos tradicionales, tal como lo menciona Storms et al., (2014).

Se determinó cantidades promedio de la biomasa y de los lípidos totales obtenidos de las tres especies de microalgas oleaginosas fueron diferentes (Tabla 2). En cuanto a la biomasa microalgal, Chlorella sp. sobresalió por presentar un mayor peso seco $(180 \pm 10$ $\mathrm{mg} / \mathrm{l})$ con respecto a Scenedesmus sp. $(127 \pm 3 \mathrm{mg} / \mathrm{l})$ y Ankystrodesmus sp. (86 $\pm 4 \mathrm{mg} / \mathrm{l})$ respectivamente,
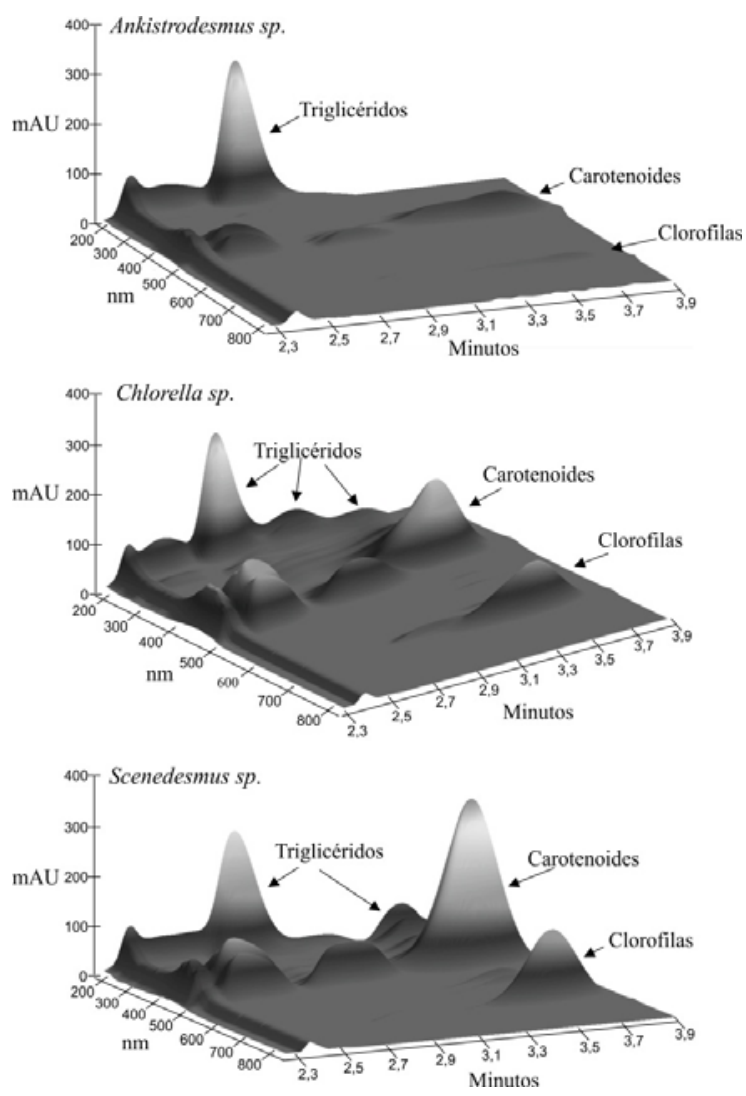

Figura 3. Cromatograma en $3 \mathrm{D}$ obtenido mediante HPLC-DAD de lípidos totales extraídos de Ankystrodesmus sp., Chlorella sp. y

Scenedesmus sp.
Tabla 2. Promedios de Biomasa y contenido de lípidos totales obtenidos de las tres especies de microalgas oleaginosas aisladas y cultivadas por triplicado.

\begin{tabular}{llll}
\hline $\begin{array}{l}\text { Especie de } \\
\text { microalga }\end{array}$ & $\begin{array}{l}\text { Biomasa en peso } \\
\text { seco }(\mathrm{mg} / \mathrm{l})\end{array}$ & $\begin{array}{l}\text { Lípidos } \\
(\%)\end{array}$ & totales \\
\hline Ankistrodesmus & $86+/-4$ & $43+/-2$ & \\
Chlorella sp. & $180+/-10$ & $22+/-3$ & \\
Scenedesmus sp. & $127+/-3$ & $28+/-3$ & \\
\hline
\end{tabular}

siendo estas diferencias estadísticamente significativas $(\mathrm{F}=163 ; \mathrm{GL}=2 ; \mathrm{P}<0.001)$. Sin embargo, respecto a los lípidos totales, los análisis nos muestran que Ankystrodesmus sp. posee un mayor contenido de estos aceites, presentando $43 \pm 2 \%$ con respecto a Scenedesmus sp. $(28 \pm 2 \%)$ y Chlorella sp. $(22 \pm 3$ $\%$ respectivamente, siendo estas diferencias estadísticamente significativas $(\mathrm{F}=71.5 ; \mathrm{GL}=2 ; \mathrm{P}<$ 0.001). Estos resultados son comparables a otra investigación que muestra un alto contenido de lípidos totales $(24.5$ a $40.3 \%)$ en una especie del género Ankistrodesmus (Jayanta et al., 2012). Sin embargo, difieren con lo reportado por Yang et al. (2012) en el caso de Chlorella sp. que presentó un contenido lipídico entre 28 y $43 \%$ en cultivo en bioreactores. Las microalgas amazónicas estudiadas presentan características apropiadas para la producción de biodiesel, en especial la especie Ankystrodesmus por presentar mayor contenido de lípidos totales. Pero, es necesario realizar más estudios que nos permitan conocer el tipo de triglicéridos que presentan cada una de ellas, ya que la mayoría de los ácidos grasos encontrados en los lípidos microalgales son moléculas de cadena recta con un número par de átomos de $\mathrm{C}$ (generalmente entre $\mathrm{C} 12$ y $\mathrm{C} 22$ ) resultado de su biosíntesis desde acetato por alfa-adición. Pueden ser saturados o insaturados (Kyle, 1991; Brown et al., 1993; Chisti, 2007; Barraza et al., 2009). Además, los ácidos grasos altamente poliinsaturados (n-3) de cadena entre C14-C24 son las moléculas ideales para la producción de metil ésteres o biodiesel a partir de microalgas (Aullon, 2010); aun así se utilizan principalmente los triacilglicéridos para la obtención de biodiesel.

Los análisis mediante HPLC-DAD revelaron que las tres especies microalgales contienen diversos compuestos lipídicos, tales como triglicéridos, pigmentos carotenoides y clorofilas que varian entre las tres especies (Figura 3). Pero, los lípidos totales extraídos de Ankystrodesmus sp., a diferencia de las otras especies evaluadas, estaban representados principalmente por triglicéridos. En contraste, Chlorella sp. y Scenedesmus sp. presentaron menor contenido de triglicéridos y mayor contenido de pigmentos. También, bajo las condiciones de separación cromatográfica se observó hasta cinco tipos de triglicéridos distinguibles por sus tiempos de retención (Figura 4). Es evidente que en las tres especies de microalgas el triglicérido predominante 


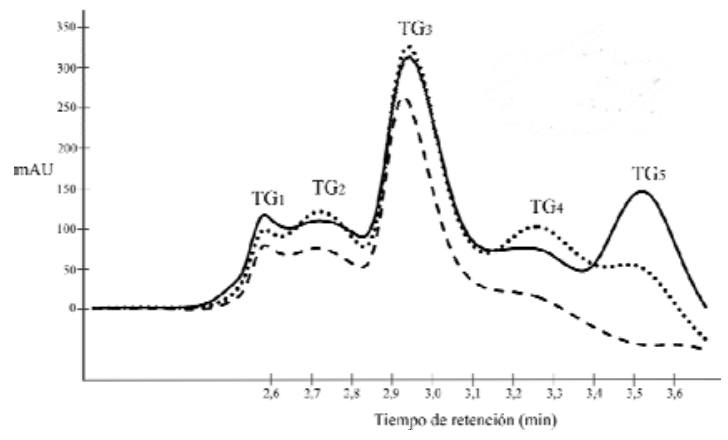

Figura 4. Cromatograma de triglicéridos presentes en los lípidos totales extraídos de la tres especies de microalgas evaluadas. Leyenda: $\mathrm{TG}_{1}=$ triglicéridos tipo $1, \mathrm{TG}_{2}=$ triglicéridos tipo 2 , $\mathrm{TG}_{3}=$ triglicéridos tipo $3, \mathrm{TG}_{4}=$ triglicéridos tipo $4, \mathrm{TG}_{5}=$ triglicéridos tipo 5 .

$\left(\mathrm{TG}_{3}\right)$ tuvo un tiempo de retención de $\sim 2.95$ minutos. Además, se evidencia que los triglicéridos de Chlorella sp. y Scenedesmus sp. muestran perfiles cromatográficos similares. Estos resultados difieren de los reportados por MacDougall et al. (2011), quienes encontraron que Botryococcus braunii, y Scenedesmus obliquus producen cantidades significativas de triglicéridos lo cual es evidente con los intensos picos en el cromatograma que tuvieron un tiempo de retención de $8-12$ minutos. En contraste, Neochloris oleoabundans produjo una alta abundancia de lípidos polares en la primera parte del cromatograma, así como picos anchos de fosfocolina en la parte posterior pero, relativamente pequeñas cantidades de TAGs. Por tanto mediante la cromatografía por HPLC-DAD se puede mejorar la selección de microalgas oleaginosas promisorias para la producción de biodiesel. Sin embargo, debido a diversos grados de eficiencias de ionización para las diferentes clases de lípidos, no se puede realizar una comparación cuantitativa entre las diferentes clases de lípidos dentro del mismo cromatograma (Molina et al., 2013). Siendo recomendable utilizar el método de Cromatografía de gases para determinar lípidos en muestras de microalgas, la determinación de lípidos a partir de análisis cualitativos y cuantitativos es crucial para identificar cepas microalgales para la producción sustentable de biodiesel. Otros métodos convencionales tales como extracción con solventes o los métodos gravimétricos han sido usados por Bligh \& Dyer (1959). En este estudio nos limitamos a conocer los lípidos totales, posteriormente se está considerando la caracterización de los tipos de triglicéridos y los ácidos grasos que lo constituyen.

\section{Conclusiones.}

Varias de las especies microalgales del río Itaya son potencialmente buenas productoras de aceites y por lo tanto promisorias para la producción de biodiesel. Asimismo, las tres especies de microalgas oleaginosas aisladas muestran diferencias en sus perfiles de crecimiento, en la cantidad de biomasa y en la cantidad y tipos de lípidos totales. Siendo Ankystrodesmus sp., la especie más interesante por tener un alto contenido de lípidos totales $(>40 \%)$ con predominancia de triglicéridos. Por tanto, es una microalga oleaginosa promisoria para la producción sustentable de biodiesel.

\section{Agradecimientos.}

Al Consejo Nacional de Ciencia Tecnología e Innovación Tecnológica (CONCYTEC) y a la Universidad Científica del Perú (UCP) por el soporte financiero. Un especial agradecimiento al Dr. J. Dylan Maddox por la revisión crítica del Abstract.

\section{Literatura citada.}

Arredondo B.O. \& Vázquez R. 1991. Aplicaciones biotecnológicas en el cultivo de microalgas. Ciencia y Desarrollo, CONACyT 27 (98): 99-111.

Aullon A.A. 2010. Biodiesel from microalgae. Tesis de grado. Royal School of Technology. Stockholm, Suecia. 92p.

Barraza C.V., Collao C., Espinoza F., Moya G. \& Torres M. 2009. Producción de biodiesel a partir de microalgas. Pontificia Universidad Católica de Valparaiso. 26p.

Barclay W., Jhonsen J., Chelf P., Nagle N. \& Roessler R. 1986. Microalgae culture collection 1986-1987. Sol Energy Res Inst. Golden, Colorado. 232-3079. 147p.

Bicudo C.E. \& Menezes M. 2006. Gêneros de Algas de Águas Continentais do Brasil - Chave para Identificação e Descrições (02 ed.). Brasil: Rima.

Bligh E.G. \& Dyer W.J. 1959. A rapid method of total lipid extraction and Purification. Can. J. Biochem. Physiol. 37: 911-917.

Brown L.M., Sprague S., Jarvis E.E., Dunahay T.G., Roessler P.G. \& Zeiler K.G. 1993. Biodiesel From Aquatic Species Project Report: FY. 38p.

Castillo C., Henao D. \& Tejeda L. 2010. Obtención y comparación de los aceites obtenidos de las microalgas Dunaliella salina y Chlorella nativa como materia prima para la producción de biodiesel. World Congress \& Exhibition ENGINEERING - Argentina.

Cobos M., Castro J.C., Del Águila J. \& Soplín M. 2012. Identificación de microalgas oleaginosas en el área de Concesión para Conservación, Cuenca alta Río Itaya, Loreto-Perú. Ciencia Amazónica. 2 (2): 162-168.

Chisti Y. 2007. Biodiesel from microalgae. Biotechnology Advances. 25: 294-306pp.

De La Mata P. 2011. Aplicabilidad de la cromatografía líquida y espectrometría vibracional para desarrollar modelos multivariantes para la detección y cuantificación de aceite de oliva en mezcla de aceites vegetales (Tesis Doctoral). Universidad de Granada, España.

Delucchi M.A. 2010. Impacts of biofuels on climate change, water use, and land use. Annals of the New York Academy of Sciences. 1195, 28-45.

Duong V.T., Li Y., Nowak E. \& Schenk P.M. 2012. Microalgae Isolation and Selection for Prospective Biodiesel Production. Energies. 5(12): 1835-1849. 
Edenhofer O., Pichs R., Sokona Y., Seyboth K., Matschoss P., Kadner S. \& Von Stechow C. 2012. Renewable Energy Sources and Climate Change Mitigation. Cambridge, United Kingdom and New York, NY, USA: Cambridge University Press.

Garibay A., Vásquez R., Sánchez M.P., Serrano L. \& Martínez A. 2009. Biodiesel a partir de microalgas. BioTecnología. 13(3), 38-61.

Georgianna D. R. \& Mayfield S.P. 2012. Exploiting diversity and synthetic biology for the production of algal biofuels. Nature, 488(7411): 329-335.

Greenspan P., Mayer E.P. \& Fowler S.D. 1985. Nile red: a selective fluorescent stain for intracellular lipid droplets. The Journal of Cell Biology. 100(3): 965-973.

Ho S.H., Chen C.H., Lee D.J. \& Chang J.S. 2011. Perspectives on microalgal $\mathrm{CO}_{2}$-emission mitigation systems - A review.Biotechnology Advances 29:189198.

Jayanta T., Mohan K. \& Bhabesh G. 2012. Growth, Total Lipid content and Fatty Acid Profile of a Native Strain of the Freshwater Oleaginous Microalgae Ankistrodesmus falcatus (Ralf) grown under Salt Stress Condition. International Research Journal of Biological Sciences, 1(18), 27-35.

Khoo H.H., Sharratt P.N., Das P., Balasubramanian R.K., Naraharisetti P.K. \& Shaik S. 2011. Life cycle energy and $\mathrm{CO}_{2}$ analysis of microalgae-to-biodiesel: preliminary results and comparisons. Bioresource Technology. 102(10): 5800-5807.

Kyle D.J. 1991. Speciality oils from Microalgae: new perspectives. In: Biotechnology of Plant Fats and Oils. J. Rattray (ed) American Oil Chemists Society. Champaign, Illinois. Cap. 8: 130-143pp.

Lam M.K. \& Lee K.T. 2012. Microalgae biofuels: A critical review of issues, problems and the way forward. Biotechnology Advances. 30(3): 673-690.

Larkum A.W.D., Ross I.L., Kruse O. \& Hankamer B. 2012. Selection, breeding and engineering of microalgae for bioenergy and biofuel production. Trends in Biotechnology. 30(4): 198-205.

Leonardos L. \& Lucas I. 2000. The nutritional value of algae grown under different culture conditions for Mytilus edulis. Larvae. Aquaculture; 182:301-315.

Loera M. \& Olguín E.J. 2009. Las microalgas oleaginosas como fuente de biodiesel: retos y oportunidades. Revista Latinoamericana de Biotecnología Ambiental y Algal. 1(1): 91-116.

López R. 2009. Caracterización fitoplanctónica, física y química de la Cocha Llanchama en los periodos hidrológicos de creciente y media vaciante. Loreto-Perú. Tesis para optar el título profesional de Biólogo. Facultad de Ciencias Biológicas. Universidad Nacional de la Amazonía Peruana, Iquitos-Perú 98p.

MacDougall K.M., McNichol J., McGinn P.J., O'Leary S.J. \& Melanson J.E. 2011. Triacylglycerol profiling of microalgae strains for biofuel feedstock by liquid chromatography-high-resolution mass spectrometry. Analytical and Bioanalytical Chemistry. 40: 2609-2616.

Menetrez M.Y. 2012. An overview of algae biofuel production and potential environmental impact. Environmental Science \& Technology. 46(13): 70737085.

Molina E., Acién F.G. \& Robles M. 2013. Downstream Processing of Cell Mass and Products. en Handbook of Microalgal Culture:Applied Phycology and Biotechnology, Second Edition.

Sánchez R. 1991. Estudio del fitoplancton en la cuenca del bajo Marañón y algunos tributarios menores en los períodos de vaciante y creciente. Tesis para optar el título profesional de Biólogo. Facultad de Ciencias Biológicas. Universidad Nacional de la Amazonía Peruana, Iquitos-Perú 63p.

Sharma K.K., Schuhmann H. \& Schenk P.M. 2012. High Lipid Induction in Microalgae for Biodiesel Production. Energies. 5(12): 1532-1553.

Sheehan J., Dunahay T., Benemann J. \& Roessler P. 1998. A Look Back at the U.S. Department of Energy's Aquatic Species Program-Biodiesel from Algae (ReporteTécnico-Científico. United States: U.S. Department of Energy's Office of Fuels Development. 328pp.

Storms Z. J., Cameron E., De La Hoz Siegler H. \& McCaffrey W. C. 2014. A Simple and Rapid Protocol for Measuring Neutral Lipids in Algal Cells Using Fluorescence. J. Vis. Exp. (87), e51441.

Veen M. \& Carrillo C. 2009. Impactos socio-económicos de la producción de biocombustibles en la amazonía peruana. Análisis de las cadenas de caña de azúcar, palma aceitera y Jatropha curcas (Primera.). SNV.

Wijffels R.H. \& Barbosa M.J. 2010. An outlook on microalgal biofuels. Science, 329(5993), 796-799.

Wu L.F., Chen P.C., Huang A.P. \& Lee C.M. 2012. The feasibility of biodiesel production by microalgae using industrial wastewater. Bioresource Technology. 113: 1418.

Yang X., Liu P. Hao, Z. Shi, J. \& Zhang S. 2012. Characterization and Identification of Freshwater Microalgal strain Toward Biofuel Production. BioResources. 7(1):686-695.

Yu X., Zhao P., He C., Li J., Tang X., Zhou J. \& Huang Z. 2012. Isolation of a novel strain of Monoraphidium sp. and characterization of its potential application as biodiesel feedstock. Bioresource Technology, 121, 256262.

Zhou W., Li Y., Min M., Hu B., Chen P. \& Ruan R. 2011. Local bioprospecting for high-lipid producing microalgal strains to be grown on concentrated municipal wastewater for biofuel production. Bioresource Technology. 102(13): 6909-6919.

\footnotetext{
${ }^{1}$ Laboratorio de Biotecnología y Bioenergética, Universidad Científica del Perú (UCP), Av. Abelardo Quiñones km 2,5, San Juan Bautista, Iquitos - Perú, Tel.: 261068-244, mcobos@ucp.edu.pe.

${ }^{2}$ Unidad Especializada de Biotecnología, Centro de Investigaciones de Recursos Naturales (CIRNA), Universidad Nacional de la Amazonía Peruana (UNAP), Pasaje Los Paujiles S/N, AAHH Nuevo San Lorenzo, San Juan Bautista, Iquitos - Perú.
} 